\title{
Bounds for sparse planar and volume arrays
}

\author{
Yann Meurisse and Jean-Pierre Delmas
}

November 2, 2021

\begin{abstract}
This correspondence improves and extends bounds on the numbers of sensors, redundancies and holes for sparse linear arrays to sparse planar and volume arrays. As an application, the efficiency of regular planar and volume arrays with redundancies but no holes is deduced. Also, examples of new redundancy and hole square arrays, found by exhaustive computer search, are given.
\end{abstract}

Index terms: sparse planar array, sparse volume array, difference base, minimum redundancy array, minimum hole array.

Finale version of CLN 99-275

paper submitted to the IEEE Transactions on Information theory as a correspondence

Département Signal et Image, Institut National des Télécommunications, 9 rue Charles Fourier, 91011 Evry Cedex, FRANCE. Fax: +33-1-6076 4433, e-mail: yann.meurisse@int-evry.fr jean-pierre.delmas@int-evry.fr. 


\section{Introduction}

When the number of antenna sensors available for an array is limited, the problem of optimum array geometry naturally arises. From the beamwidth and the sidelobe level of the associated beampattern [1] or from the direction of arrival (DOA) estimation accuracy [2] point of view, array configurations known as linear minimum redundancy (MR) arrays or linear minimum hole (MH) arrays (also called optimum nonredundant arrays) are often proposed. Linear MR arrays have been extensively studied; see [3] and [4] and the references therein. In particular, much attention has been given to bounds on the ratio $M^{2} / A$ [4],[5] where $M$ and $A$ denote respectively the number of sensors and the aperture of the linear array. Linear MH arrays were considered in [3] and [6]. Whereas specific structures were designed to optimize some performance criteria (e.g. [7] for DOA algorithms with DOA prior information and [1] for beampatterns with various sidelobe level/beamwidth tradeoffs); redundancy and hole concepts do not embrace any such optimality criterion directly. Thus, the MR and MH arrays are more easily applicable to a wider range of problems, and these structures achieve an efficient tradeoff between beampattern and DOA estimation performance.

Contrary to the sparse linear arrays, few contributions have been devoted to sparse planar and volume arrays (note that the planar array retains side ambiguity resolved by a volume array). The notions of MR and $\mathrm{MH}$ arrays can be extended to these arrays because the spatial covariance matrix, associated with equally spaced arrays, exhibits a Toeplitz, block-Toeplitz structure for uncorrelated sources. Some structures of square and cubic redundancy arrays were studied by Pumphrey [8]. However, as discussed in [9], the computation of MR and MH arrays for the two-dimensional case is much more involved than that for the one-dimensional case. Thus, it is of importance to have bounds to be able to qualify the efficiency of not necessarily MR or MH planar and volume structures.

Section 2 improves and extends bounds on the numbers of sensors, redundancies and holes for the sparse linear arrays given by [3] to sparse planar and volume arrays. As an application, the efficiency of regular planar and volume arrays with redundancies but no holes is deduced. Also, examples of new redundancy and hole square arrays given by exhaustive computer search are shown in Appendix.

\section{Bounds for arrays with redundancies and holes}

Consider a volume array $\mathcal{A}$ made of $M$ sensors lying on the marks of a Cartesian grid ${ }^{1}$. The sensor spacings on this grid are integer multiples of some fundamental distance (usually the half wavelength of the incident radiation), and thus the sensor separations can be represented by these integers. Based on the assumption that one is primarily interested in how an array samples the spatial covariance function, which is a function only of the separation between the points (for uncorrelated sources), the useful notion of coarray was introduced [10]. It refers to the set of points at which the spatial covariance function can be estimated with that array. This coarray $\mathcal{D}$ is represented with a set of vectors $\mathbf{d}$ called lags

$$
\mathcal{D}=\left\{\mathbf{d}_{i j}=\mathbf{s}_{i}-\mathbf{s}_{j}\right\} \quad i, j=1,2, \ldots, M
$$

where $\mathbf{s}_{i}=\left(x_{i}, y_{i}, z_{i}\right)^{T}$ is the location of the $i$ th sensor $\left(x_{i}, y_{i}\right.$ and $z_{i}$ are integers). Denote by

$$
\left(A_{x}, A_{y}, A_{z}\right)=\left(\max \left(x_{i}-x_{j}\right), \max \left(y_{i}-y_{j}\right), \max \left(z_{i}-z_{j}\right)\right)
$$

the apertures of this array. Linear and planar arrays are considered as particular cases of volume arrays, i.e., $A_{y}=A_{z}=0$ and $A_{z}=0$ for respectively linear and planar arrays. With these definitions, we recall that if the array has more than one pair of sensors separated by the same lag $\mathbf{d}$, these pairs produce redundant estimates of the covariance function at that lag. In this case, the coarray of that array is said to have redundancies. The number of these redundancies excluding the lag $\mathbf{0}$ is denoted by $R$. If there is no pair of sensors separated by some lag whose components are all smaller than the associated apertures of the array, the array is said to have a hole in its coarray at that location. The number of these holes is denoted by $H$. If $E$ is the number of distinct lags of the coarray (including lag $\mathbf{0}$ ), the number $M^{2}$ of lags $\mathbf{d}_{i j}$ of the coarray is composed of $E$ lags appearing at least one time and of $R+M-1$ strictly redundant lags, so $M^{2}=E+R+(M-1)$. Each of the $\left(2 A_{x}+1\right)\left(2 A_{y}+1\right)\left(2 A_{z}+1\right)$ lags of the rectangular parallelepiped associated full array appears either at least one time or not at all, so $E+H=\left(2 A_{x}+1\right)\left(2 A_{y}+1\right)\left(2 A_{z}+1\right)$. Consequently the apertures, the numbers of sensors,

\footnotetext{
${ }^{1}$ It is possible to consider other kind of grid as Haubrich [10] did, who considered sensors on an isometric or equilateral triangle grid and found perfect planar arrays in this way.
} 
redundancies and holes are related by

$$
\left(2 A_{x}+1\right)\left(2 A_{y}+1\right)\left(2 A_{z}+1\right)=\left(M^{2}-M+1\right)+H-R .
$$

To eliminate the apertures in this relation and derive a general relation between the numbers of sensors, redundancies and holes, we introduce a new function associated with its autocorrelation function that enables us to improve and to extend directly the linear array bounds by [3] to planar and volume arrays. Let $\chi_{\mathcal{A}}: \mathcal{Z}^{3} \rightarrow\{0,1\}$ be the characteristic function of the array, i.e. $\chi_{\mathcal{A}}(\mathbf{s})=1$ if a sensor is in position $\mathbf{s}$ and $\chi_{\mathcal{A}}(\mathbf{s})=0$ elsewhere. The number of times the lag $\mathbf{d}$ is present in the sparse array defines the autocorrelation function $\Lambda(\mathbf{d})$ of $\chi_{\mathcal{A}}: \mathcal{Z}^{3} \rightarrow \mathcal{N}$. With these definitions, the following result is proved.

Result 1 For a given number of sensors, the numbers of holes and redundancies that must be present in the linear, planar or volume array satisfy the relation: ${ }^{2}$

$$
R\left(3 \pi+2\left(1+\alpha_{A}\right)\right)+H\left(3 \pi-2\left(1+\alpha_{A}\right)\right) \geq 2\left(1+\alpha_{A}\right) M^{2}-(M-1)\left(3 \pi+2\left(1+\alpha_{A}\right)\right)
$$

where $\alpha_{A}$ is given in the Appendix ${ }^{3}$.

Proof Let $\chi_{\overline{\mathcal{D}}}$ be the characteristic function of the coarray associated with the fully populated array of apertures $\left(A_{x}, A_{y}, A_{z}\right)$ and $\chi_{\mathbf{0}}: \mathcal{Z}^{3} \rightarrow\{0,1\}$ that of lag $\mathbf{0}$ whose Fourier transforms are respectively $\frac{\sin \pi\left(2 A_{x}+1\right) f_{x}}{\sin \pi f_{x}} \frac{\sin \pi\left(2 A_{y}+1\right) f_{y}}{\sin \pi f_{y}} \frac{\sin \pi\left(2 A_{z}+1\right) f_{z}}{\sin \pi f_{z}}$ and 1 .

As $\Lambda(\mathbf{d})$ is the number of times the lag $\mathbf{d}$ appears in the array $\mathcal{A}$, the difference

$$
\epsilon(\mathbf{d}) \stackrel{\text { def }}{=} \Lambda(\mathbf{d})-\chi_{\overline{\mathcal{D}}}(\mathbf{d})-(M-1) \chi_{\mathbf{0}}(\mathbf{d})
$$

satisfies for $\mathbf{d}$ in $\overline{\mathcal{D}}: \epsilon(\mathbf{d})=-1$ if $\mathbf{d}$ is a hole, $\epsilon(\mathbf{d})$ is equal to the number of redundancies of that lag $\mathbf{d}$ if $\mathbf{d}$ is in $\mathcal{D}$, except for $\mathbf{d}=\mathbf{0}$ in which case $\epsilon(\mathbf{d})=\mathbf{0}$. Consequently $\sum_{\mathbf{d} \in \overline{\mathcal{D}}}|\epsilon(\mathbf{d})|=H+R$. As the Fourier transform $E(\mathbf{f})$ of the even real function $\epsilon(\mathbf{d})$ is real, $E(\mathbf{f}) \leq|E(\mathbf{f})| \leq \sum_{\mathbf{d} \in \overline{\mathcal{D}}}|\epsilon(\mathbf{d})|=H+R$. Therefore, taking the Fourier transform of (2.3) and noting that the Fourier transform $L(\mathbf{f})$ of the autocorrelation function $\Lambda(\mathbf{d})$ is real non negative, there holds for all $\mathbf{f}$ :

$$
-\frac{\sin \pi\left(2 A_{x}+1\right) f_{x}}{\sin \pi f_{x}} \frac{\sin \pi\left(2 A_{y}+1\right) f_{y}}{\sin \pi f_{y}} \frac{\sin \pi\left(2 A_{z}+1\right) f_{z}}{\sin \pi f_{z}}-(M-1) \leq H+R-L(\mathbf{f}) \leq H+R .
$$

After simple manipulations detailed in the Appendix, we obtain:

$$
\frac{2}{3 \pi}\left(1+\alpha_{A}\right)\left(2 A_{x}+1\right)\left(2 A_{y}+1\right)\left(2 A_{z}+1\right)-(M-1) \leq H+R .
$$

Finally, substituting (2.1) in this expression yields (2.2).

Result 1, identical to [3, rel. (19)] except for the presence of $\alpha_{A}$, improves the classic one-dimensional bound [1], and extends it to two- and three-dimensional arrays. Surprisingly, this result is invariant with respect to the dimensionality of the array. Putting respectively $H=0$ and $R=0$ in (2.2) gives lower bounds on $R$ and $H$ for respectively redundancy arrays and hole arrays. Whatever the dimensionality of the array may be, we have, for redundancy arrays $R \geq \frac{2\left(1+\alpha_{A}\right) M^{2}}{3 \pi+2\left(1+\alpha_{A}\right)}-(M-1)$ and for hole arrays $H \geq \frac{2\left(1+\alpha_{A}\right) M^{2}}{3 \pi-2\left(1+\alpha_{A}\right)}-(M-1) \frac{3 \pi+2\left(1+\alpha_{A}\right)}{3 \pi-2\left(1+\alpha_{A}\right)}$. Thus, for large arrays, the lower bounds provided by [3, rel. (20) and (21)] are approximatively multiplied by 1.0237 .

Concerning the bounds on $M$, result 1 implies the following:

1. For perfect arrays, i.e., arrays with no redundancy or hole $(R=0 ; H=0)$, result 1 implies $2\left(M^{2}-M+1\right)-$ $3 \pi(M-1) \leq 0$, therefore $M \in\{2,3,4\}$. Thus from $(2.1), M^{2}-M+1=\left(2 A_{x}+1\right)\left(2 A_{y}+1\right)\left(2 A_{z}+1\right)=3,7$ or 13. Since the product $\left(2 A_{x}+1\right)\left(2 A_{y}+1\right)\left(2 A_{z}+1\right)$ is prime, the only, non trivial solutions are:

$$
M=3 ; A_{x}=3 ; A_{y}=A_{z}=0 \quad \text { and } \quad M=4 ; A_{x}=6 ; A_{y}=A_{z}=0 .
$$

These are the well-known linear perfect arrays [3] and we prove that the only perfect arrays are linear.

\footnotetext{
${ }^{2}$ Note that in our formulation, lags $\mathbf{d}$ are in $\mathcal{Z}^{3}$, so our definitions of $H$ and $R$ differ from those of [3, rel. 19] in the linear case by the multiplicative factor 2 .

${ }^{3}$ In particular it is shown in the Appendix that $\alpha_{A} \ll 1$ and that $\alpha_{A} \approx 0.0237$ for apertures $\geq 6$.
} 
2. For redundancy arrays [3], i.e., arrays with no hole $(H=0)$, result 1 and (2.1) yield:

$$
M^{2} \geq\left(2 A_{x}+1\right)\left(2 A_{y}+1\right)\left(2 A_{z}+1\right)\left(1+\frac{2\left(1+\alpha_{A}\right)}{3 \pi}\right),
$$

which for $A_{y}=A_{z}=0$ gives a tighter lower bound on $M$ than [3, rel. (17)]. However, because of round-off effect, the improvement provided by (2.5) in the one-dimensional case is not regular and occurs for only certain values of $A$. For example, for LMR arrays, $A=69$ is the lowest value of $\mathrm{A}$ for which the lower bound on $M$ provided by [3, rel. (17)] and by (2.5) would be different (respectively $M \geq 13$ and $M \geq 14$ ).

3. For hole arrays [3], i.e. arrays with no redundancy $(R=0)$, result 1 and (2.1) yield:

$$
(M-1)^{2} \leq\left(2 A_{x}+1\right)\left(2 A_{y}+1\right)\left(2 A_{z}+1\right)\left(1-\frac{2\left(1+\alpha_{A}\right)}{3 \pi}\right)-1,
$$

which for $A_{y}=A_{z}=0$ gives a tighter upper bound on $M$ than [3, rel. (26)]. But, as previously, the improvement in the one-dimensional case is not regular and, for example, for LMH arrays, $A=51$ is the lowest value of A for which the upper bound on $M$ provided by [3, rel. (26)] and by (2.6) would be different (respectively $M \leq 10$ and $M \leq 9$ ).

\section{Design of sparse linear and volume arrays}

To build efficient square and cubic redundancy arrays, two regular structures are known. The first ones, referred to by Pumphrey[8] as "cross product" (CP) arrays, are constructed from respectively two or three identical linear MR arrays. A square cross product array for example has an sensor at $\{i, j\}$ if the linear array it is constructed from has an sensor at $\{i\}$ and one at $\{j\}$ (see Fig.2). Note that these structures can be extended to nonidentical linear MR arrays. As linear MR arrays are built for any $M$, their optimal aperture $A$ is a function $A_{M}$ of $M$. So associated cross product arrays are defined only for these apertures $A_{M}$, for which the number of sensors is $M^{2}$ or $M^{3}$. As for linear MR arrays [5],[4], $2.434 \leq \lim _{M \rightarrow \infty} \frac{M^{2}}{A_{M}} \leq 3.348$, therefore these cross product structures satisfy respectively for the square and cubic arrays with $M$ sensors,

$$
2.434 \leq \lim _{M \rightarrow \infty} \frac{M}{A_{M}} \leq 3.348 \quad \text { and } \quad 2.434 \leq \lim _{M \rightarrow \infty} \frac{M^{2 / 3}}{A_{M}} \leq 3.348 .
$$

Compared to (2.5), which gives for respectively linear, square and cubic redundancy arrays $M^{2} \geq 2.434 A+1.217$, $M \geq 2.207 A+1.103$ and $M^{2 / 3} \geq 2.135 A+1.068$, these cross product redundancy arrays are potentially efficient. However, we note that the difference between the number of sensors given by the lower bound (2.5) and the one given by (3.1) increases with the dimensionality of the array. A second regular structure was proposed by Greene-Wood (GW) [11] for square arrays. The sensor location $(i, j, k)$ of such an array of aperture $A$ verifies : $i=0$ or $j=0$ or $k=0$ or $i=j=k=2, \cdots, A$ ( $k=0$ for square array cf. Fig.2). It gives respectively $M=3 A$ and $M=A(3 A+4)$ for square and cubic arrays ${ }^{4}$. Compared to $(2.5)$, the Greene-Wood square array is a potentially efficient redundancy array contrary to the Greene-Wood cubic array. Naturally all these structures are not necessarily MR. Table 1 exhibits, by exhaustive computer search, the number of MR and MH square arrays for apertures up to 6 ( 7 for MH square arrays), two arrays being considered different if none of them can be deduced from the other by an elementary transformation. We find that these arrays are not generally unique. However, Greene-Wood or cross product MR arrays exist for each of these apertures (except for $A=2$ ).

\footnotetext{
${ }^{4}$ Note that a more efficient cubic structure can be obtained by piling up identical GW square arrays for which the number of sensors is $M=3 A(A+1)$
} 


\begin{tabular}{|c|c|c|c|c|c|c|c|c|}
\hline \multirow{3}{*}{ Aperture } & \multicolumn{5}{|c|}{ square MR array } & \multicolumn{3}{|c|}{ square MH array } \\
\hline & \multirow[t]{2}{*}{$M_{\min }$} & \multirow[t]{2}{*}{ LB } & \multicolumn{3}{|c|}{ Number of configurations } & \multirow[t]{2}{*}{$M_{\max }$} & \multirow[t]{2}{*}{ UB } & \multirow{2}{*}{$\begin{array}{l}\text { Number of } \\
\text { configurations }\end{array}$} \\
\hline & & & Total & GW & CP & & & \\
\hline 2 & 7 & 6 & 2 & & & 5 & 5 & 1 \\
\hline 3 & 9 & 8 & 4 & 1 & 1 & 6 & 7 & 36 \\
\hline 4 & 12 & 10 & 4 & 1 & & 8 & 8 & 4 \\
\hline 5 & 15 & 13 & 83 & 1 & & 9 & 10 & 347 \\
\hline 6 & 16 & 15 & $\geq 1$ & & 1 & 11 & 12 & 1 \\
\hline 7 & & 17 & & & & 12 & 14 & 113 \\
\hline
\end{tabular}

Table 1 Numbers of sensors and configurations of square MR [resp. MH] arrays obtained by exhaustive computer search compared with lower bound (LB) [resp. upper bound (UB)], versus aperture.

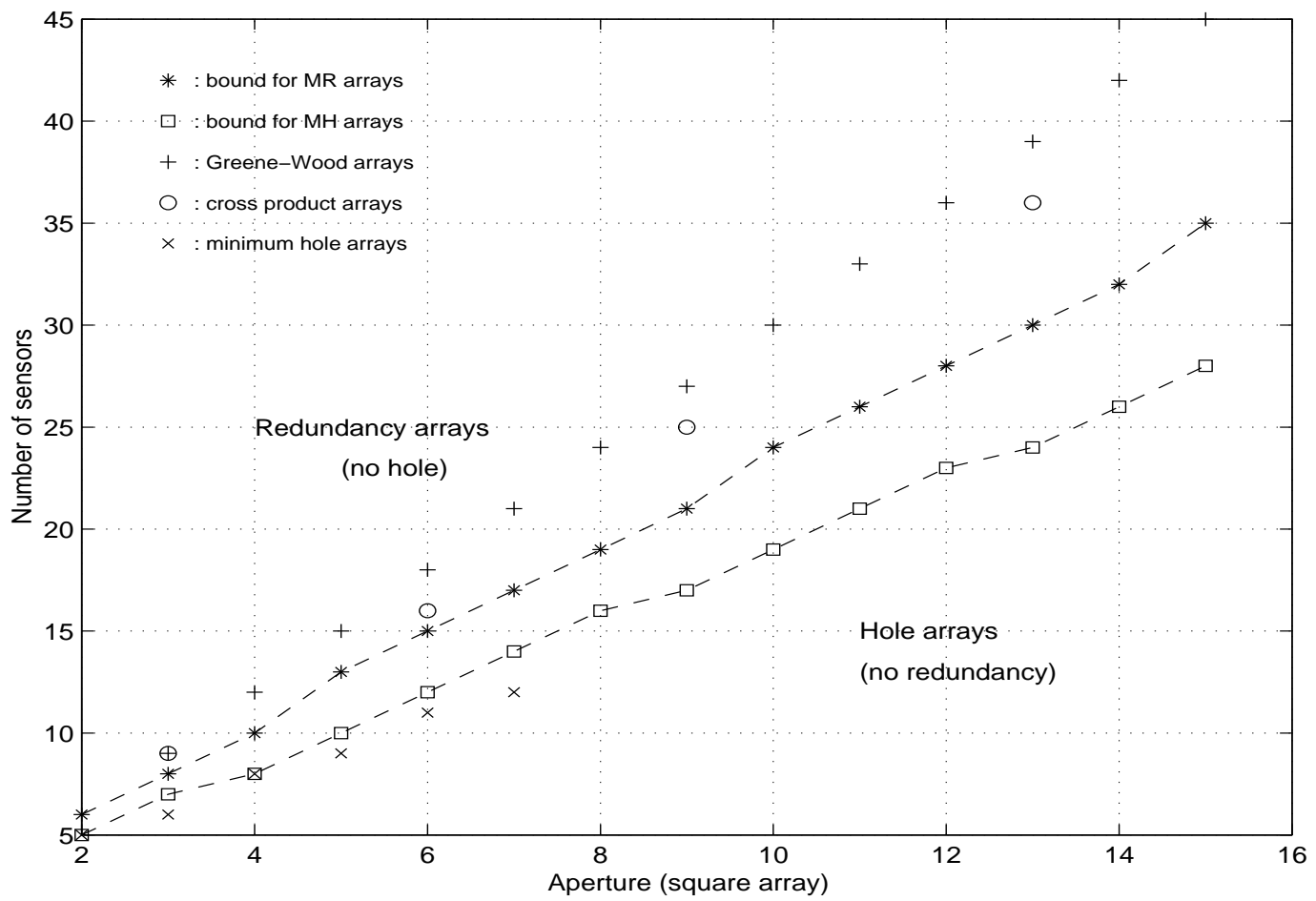

Fig.1 Numbers of sensors (obtained by exhaustive computer search and bounds (2.5),(2.6)) versus aperture of square array.

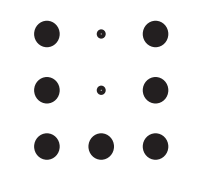

$\mathrm{M}=7 \mathrm{R}=18$

$\mathrm{M}=9 \quad \mathrm{R}=24$ (cross product array)

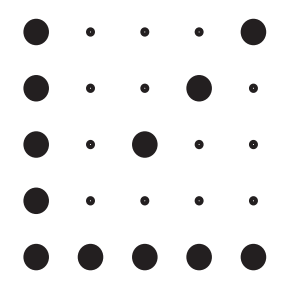

$\mathrm{M}=12 \quad \mathrm{R}=52$

(Greene-Wood array)

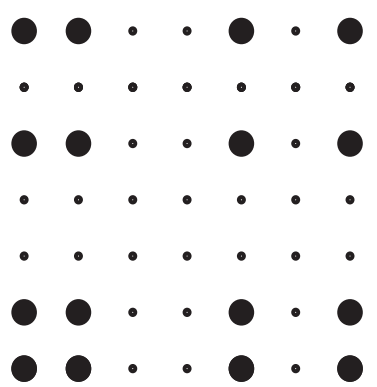

$M=16 \quad R=72$ (cross product array)

Fig.2 Examples of square MR arrays. 


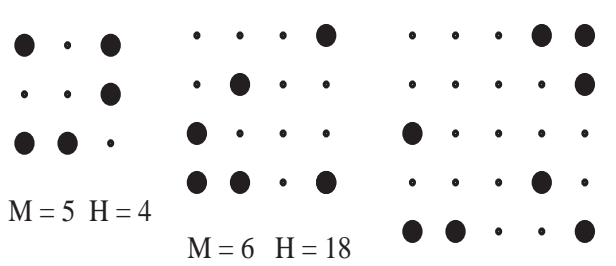

$M=8 \quad H=32$

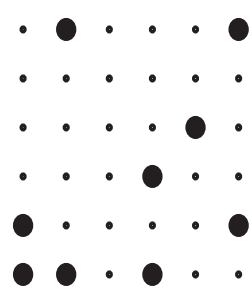

$\mathrm{M}=9 \quad \mathrm{H}=48$

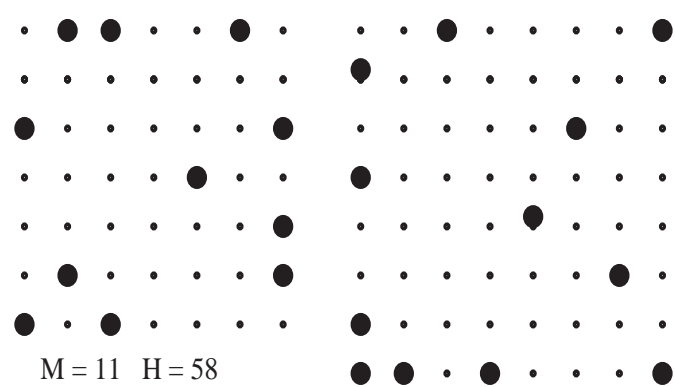

$\mathrm{M}=12 \quad \mathrm{H}=92$

Fig.3 Examples of square MH arrays.

\section{Conclusion}

A general formulation has enabled us to consider the notion of minimum hole and minimum redundancy arrays regardless of the dimensionality of the array. Thanks to this approach, tighter bounds have been given on the numbers of sensors, redundancies and holes for the linear arrays and similar bounds have been proposed for planar and volume arrays. As an application, the efficiency of regular planar and volume arrays with redundancies but no holes has been deduced. The number of sensors and configurations of square MR and MH arrays obtained by exhaustive computer search has been given. An example of square MR and MH array for each aperture is exhibited up to aperture 7. Finally, we note that designing simple regular structures of efficient hole arrays, or deducing hole planar and volume arrays from linear hole arrays, still presents a number of obstacles.

\section{A Proof of (2.4)}

Let $s(\mathbf{A}, \mathbf{f})$ denote the expression $-\frac{\sin \pi\left(2 A_{x}+1\right) f_{x}}{\sin \pi f_{x}} \frac{\sin \pi\left(2 A_{y}+1\right) f_{y}}{\sin \pi f_{y}} \frac{\sin \pi\left(2 A_{z}+1\right) f_{z}}{\sin \pi f_{z}}$. Because explicit calculation of $\max _{\mathbf{f}} s(\mathbf{A}, \mathbf{f})$ is complicated, we shall focus on two slightly smaller values. First, as $\max _{x}\left(-\frac{\sin x}{x}\right)=\frac{2}{3 \pi}\left(1+\alpha^{\prime}\right)$ with $\alpha^{\prime}=0.0237$ and $\sin x<x$ for $x>0$, direct manipulations imply:

$$
\frac{2}{3 \pi}\left(1+\alpha^{\prime}\right)\left(2 A_{x}+1\right)\left(2 A_{y}+1\right)\left(2 A_{z}+1\right) \leq \max _{\mathbf{f}} s(\mathbf{A}, \mathbf{f}) .
$$

Second, suppose $A_{x}=\min \left(A_{x}, A_{y}, A_{z}\right)$ without loss of generality. For $\mathbf{f}_{0} \stackrel{\text { def }}{=}\left(f_{0}, 0,0\right), f_{0} \stackrel{\text { def }}{=} \frac{3}{2\left(2 A_{x}+1\right)}$ :

$$
-\frac{\sin \pi\left(2 A_{x}+1\right) f_{0}}{\sin \pi f_{0}}\left(2 A_{y}+1\right)\left(2 A_{z}+1\right)=s\left(\mathbf{A}, \mathbf{f}_{0}\right) \leq \max _{\mathbf{f}} s(\mathbf{A}, \mathbf{f}) .
$$

Using the classical relation between the periodic Fourier transform of a sequence and the Fourier transform of the associated analog waveform,

$$
\begin{aligned}
-\frac{\sin \pi\left(2 A_{x}+1\right) f_{0}}{\sin \pi f_{0}} & =-\sum_{k=-\infty}^{+\infty} \frac{\sin \left(\pi\left(2 A_{x}+1\right)\left(f_{0}-k\right)\right)}{\pi\left(f_{0}-k\right)}=-\frac{\sin \pi\left(2 A_{x}+1\right) f_{0}}{\pi} \sum_{k=-\infty}^{+\infty} \frac{(-1)^{k}}{f_{0}-k} \\
& =-\frac{\sin \pi\left(2 A_{x}+1\right) f_{0}}{\pi}\left(\frac{1}{f_{0}}+2 f_{0} \sum_{k=1}^{+\infty} \frac{(-1)^{k}}{f_{0}{ }^{2}-k^{2}}\right) \\
& =\frac{2}{3 \pi}\left(2 A_{x}+1\right)\left(1-\frac{9}{2} \sum_{k=1}^{+\infty} \frac{(-1)^{k}}{k^{2}\left(2 A_{x}+1\right)^{2}-\frac{9}{4}}\right) \stackrel{\text { def }}{=} \frac{2}{3 \pi}\left(2 A_{x}+1\right)\left(1+\alpha_{A}^{\prime \prime}\right) .
\end{aligned}
$$

Therefore

$$
\frac{2}{3 \pi}\left(1+\alpha_{A}^{\prime \prime}\right)\left(2 A_{x}+1\right)\left(2 A_{y}+1\right)\left(2 A_{z}+1\right) \leq \max _{\mathbf{f}} s(\mathbf{A}, \mathbf{f}) .
$$

Combining these two values, (2.4) holds with $\alpha_{A} \stackrel{\text { def }}{=} \max \left(\alpha^{\prime}, \alpha_{A}^{\prime \prime}\right)$. We note that $\alpha_{A}^{\prime \prime}$ is decreasing in $A_{x}$ and a sharp examination of $\alpha_{A}^{\prime \prime}$ shows that $\alpha_{A}=\alpha_{A}^{\prime \prime}$ for $A_{x}<6$ (e.g. $\alpha_{3} \approx 0.0797$ ) and $\alpha_{A}=\alpha^{\prime} \approx 0.0237$ for $A_{x} \geq 6$. 


\section{References}

[1] S. De Graff, D.H. Johnson, "Optimal linear arrays for narrow-band beamforming," in Proc. ICASSP-84, pp. 40.8.1-4, 1984.

[2] Y.I. Abramovich, D.A. Gray, A.Y. Gorokhov and N.K. Spencer, "Comparison of DOA estimation performance for various types of sparse antenna array geometries," in Proc. EUSIPCO-96, Trieste, pp. 915-918, 1996.

[3] D.A. Linebarger, I.H. Sudborough and I.G. Tollis, "Difference bases and sparse sensor arrays," IEEE Trans. on Information Theory, vol. 39, no. 2, pp. 716-721, March 1993.

[4] S.U. Pillai, Array Signal processing, Springer Verlag, 1988.

[5] J. Leech, "On the representation of 1, 2, .., n by differences," J. London Math. Soc., vol. 31, pp. 160-169, 1956.

[6] E. Vertatschitsch, S. Haykin, "Nonredundant array," Proc. IEEE, vol. 74, pp. 217, January 1986.

[7] X. Huang, J.P. Reilly and M. Wong, "Optimal design of linear array of sensors," in Proc. ICASSP-91, Toronto, pp. 1405-1408, 1991.

[8] H.C. Pumphrey, "Design of sparse arrays in one, two, and three dimensions," J. Acoust. Soc. Am, vol. 93, no. 3, pp. 1620-1628, March 1993.

[9] S. Haykin, J.P. Reilly, V. Kezys and E. Vertatschitsch, "Some aspects of array signal processing," IEE Proceedings-F, vol. 139, no. 1, pp. 1-26, February 1992.

[10] R. A. Haubrich, "Array design," Bull. Seismol. Soc. Am, vol. 58, pp. 977-991, 1968.

[11] C.R. Greene, R.C. Wood, "Sparse array performance," J. Acoust. Soc. Am., Vol. 63, pp. 1866-1872, 1978.

Yann Meurisse received the Engineer degree in Telecommunications from the Institut National des Télécommunications (INT), Evry, France in 1983 and the Diplome d'Etudes Approfondies in Automatic Control and Signal Processing from University Paris XI Orsay in 1987.

Since 1985, he has been with the Institut National des Télécommunications, where he is currently an Assistant Professor in the Signal and Image Processing Department. His research interests include antenna array processing.

Jean Pierre Delmas was born in France in 1950. He received the Engineer Degree from the Ecole Centrale de Lyon, France in 1973 and the Certificat d'Etudes Supérieures from the Ecole Nationale Supérieure des Télécommunications (ENST), Paris, France in 1982.

Since 1980, he has been with the Institut National des Télécommunications, where he is currently an Associate Professor in the Signal and Image Processing Department. His research interests are in statistical signal processing. 